\title{
Comparison of the Thin Convex Probe Endobronchial Ultrasound Bronchoscope to Standard Endobronchial Ultrasound and Flexible Bronchoscope-A Cadaveric Study
}

\author{
Sean P Callahan, ${ }^{1}$ Nichole T Tanner, ${ }^{1,2}$ Alexander Chen, ${ }^{3}$ Thierry Bacro, ${ }^{4}$ Gerard A Silvestri ${ }^{1}$ and Nicholas J Pastis ${ }^{1}$ \\ 1. Department of Pulmonary, Critical Care and Sleep Medicine, Medical University of South Carolina, Charleston, SC, US; 2. Ralph H Johnson \\ Veterans Affairs Hospital, Charleston, SC, US; 3. Division of Pulmonary and Critical Care Medicine, Washington University School of Medicine, \\ St Louis, MO, US; 4. Department of Regenerative Medicine and Cell Biology, Medical University of South Carolina, Charleston, SC, US
}

DOI: https://doi.org/10.17925/USRPD.2017.12.02.33

\section{Keywords}

Endobronchial ultrasound, convex probe, thin convex probe, bronchoscopy, cadaver

\footnotetext{
Disclosure: Nichole T Tanner is currently receiving grant funding and consulting fees from Olympus America, Inc. and Cook Inc. Alexander Chen is currently receiving research funding and consulting fees from Olympus America, Inc. Gerard A Silvestri and Nicholas I Pastis are currently receiving grant funding from Olympus America, Inc. Sean P Callahan and Thierry Bacro have no conflict of interest or source funding to declare. The abstract was presented at Advancements in Lung Cancer Diagnostics and Treatment Session; American College of Chest Physicians Chest Annual Meeting 2016; Chest. 2016;150 (Suppl. 4):979A.

Compliance with Ethics: All procedures were performed under the guidance of the institutional review board of the Medical University of South Carolina (Pro\#37817).

Authorship: All named authors meet the International Committee of Medical Journal Editors (ICMJE) criteria for authorship of this manuscript, take responsibility for the integrity of the work as a whole, and have given final approval to the version to be published.

open Access: This article is published under the Creative Commons Attribution Noncommercial License, which permits any noncommercial use, distribution, adaptation, and reproduction provided the original author(s) and source are given appropriate credit.

Received: October 17, 2016

Accepted: December 13, 2016

Citation: US Respiratory \& Pulmonary Diseases, 2017;2(1):33-6

Corresponding Author: Sean P Callahan, Medical University of South Carolina, 96 Jonathan Lucas Street, CSB 816, MSC 630, Charleston, South Carolina 29425, US. E: callahse@musc.edu
}

Support: The study was supported by a grant from Olympus America, Inc.
Assessment of peripheral pulmonary nodules and mediastinal lymph nodes is critical in the diagnosis and staging of lung cancer, which is the leading cause of cancer death worldwide., ${ }^{1,2}$ Endobronchial ultrasound (EBUS) continues to evolve, allowing clinicians the ability to evaluate peripheral and central pulmonary lesions as well as various layers of bronchi. Current EBUS bronchoscopes are larger and less maneuverable than a standard bronchoscope, which limits their ability to access certain regions of the lung.

Convex probe endobronchial ultrasound (CP-EBUS) with transbronchial needle aspiration (TBNA) is used to sample mediastinal and hilar lymph nodes and is considered a first-line test for mediastinal staging of lung cancer. ${ }^{3}$ It is an effective, safe, and minimally invasive procedure, which can also diagnose sarcoidosis and lymphoma. ${ }^{4,5}$ While CP-EBUS provides access to mediastinal nodes and centrally located pulmonary lesions, it is limited in its ability to sample lesions located more distal to the central airways. A $35^{\circ}$ forward oblique direction of view, the diameter of the scope, and the inability to flex the scope at an acute angle make it particularly difficult for the CP-EBUS bronchoscope to navigate into the upper lobes and the narrow right middle lobe (RML) orifice. ${ }^{6}$ An alternative to the CP-EBUS is radial EBUS (r-EBUS), which can reach the periphery of the lung; however, lack of 'real-time' ultrasound during needle puncture is a limiting factor.

These limitations led to the development of the thin CP-EBUS (TCP-EBUS) (OLYMPUS MEDICAL SYSTEMS CORP, Tokyo, Japan) to perform real-time 
Table 1: Comparison of the thin convex probe endobronchial ultrasound, the current convex probe endobronchial ultrasound and the standard flexible bronchoscope

\begin{tabular}{|l|l|l|l|}
\hline $\begin{array}{l}\text { Bronchoscope } \\
\text { Specifications }\end{array}$ & $\begin{array}{l}\text { CP-EBUS } \\
\text { Bronchoscope }\end{array}$ & $\begin{array}{l}\text { Thin EBUS } \\
\text { Bronchoscope }\end{array}$ & $\begin{array}{l}\text { Standard } \\
\text { Bronchoscope }\end{array}$ \\
\hline Working length (mm) & 600 & 600 & 600 \\
\hline Direction of view (deg.) & 35 & 20 & 0 \\
\hline Insertion Tube diameter (mm) & 6.3 & 5.7 & 5.5 \\
\hline Distal Tip (mm) & 6.9 & 5.9 & 5.1 \\
\hline Angle of flexion (up) (deg.) & 120 & 170 & 210 \\
\hline
\end{tabular}

CP-EBUS = convex probe endobronchial ultrasound; $E B U S=$ endobronchial ultrasound

Figure 1: The appearance of both the thin convex probe endobronchial ultrasound to the current convex probe endobronchial ultrasound

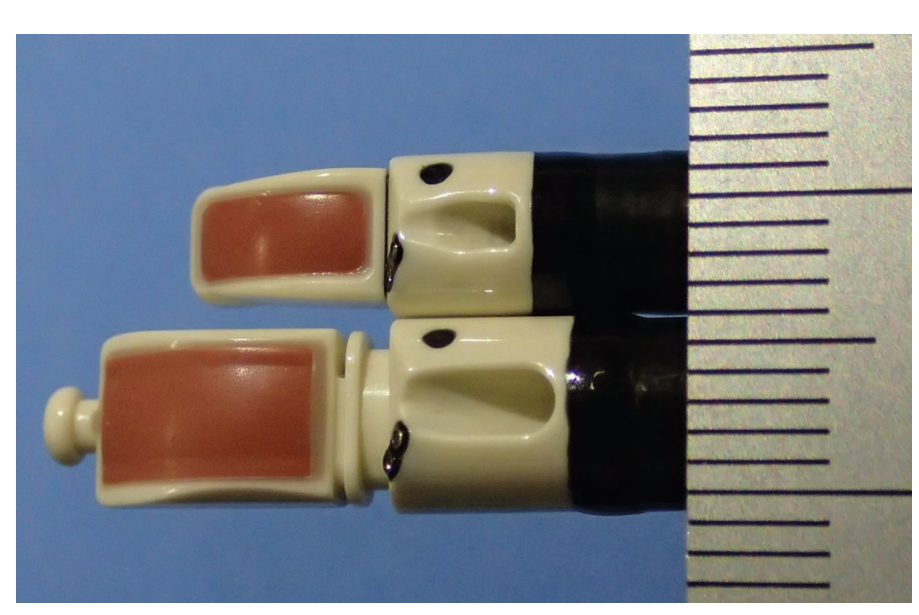

EBUS-TBNA at and around segmental bronchi. The TCP-EBUS scope has a more favorable forward oblique direction of view $\left(20^{\circ}\right)$, a smaller diameter distal tip, and greater range of angulation than traditional CP-EBUS. This technology was previously tested in a porcine model and demonstrated improved accessibility to peripheral bronchi and capability of sampling segmental lymph nodes.? A recent study assessed the effectiveness of performing both an airway inspection and TBNA of mediastinal, hilar and proximal parenchymal lymph nodes using a similar hybrid thin EBUS model. In this randomized, controlled human trial, a modified CP-EBUS bronchoscope with smaller diameter distal tip, wider angle of flexion and better direction of view, could provide better visualization of lung segments and allow for fewer bronchoscopes when performing a myriad of procedures. ${ }^{8}$

We undertook this study to further evaluate the TCP-EBUS prototype in a cadaveric model to objectively document the endobronchial territory it can access and compare it to that of the current CP-EBUS and standard sized diagnostic flexible bronchoscope.

\section{Methods}

Two cadavers of differing sizes, one male and female, embalmed with Carolina Perfect Solution ${ }^{\circledR}$ (Carolina Biological Supply Co., Burlington, NC) were secured through the Medical University of South Carolina (MUSC) Center for Anatomical Studies and Education and were used for the purposes of this experiment. Cricothyrotomy with $8 \mathrm{~mm}$ endotracheal tube placement was performed on both to access the airways. Bag mask ventilation was then used to re-inflate the lungs. A standard flexible bronchoscope was introduced and therapeutic suction of secretions performed.

Three separate bronchoscopes were used for this experiment: standard bronchoscope (BF-H190, OLYMPUS MEDICAL SYSTEMS CORP, Tokyo, Japan), linear CP-EBUS bronchoscope (BF-UC180F OLYMPUS MEDICAL SYSTEMS CORP, Tokyo, Japan), and the TCP-EBUS bronchoscope (BF-Y0055, OLYMPUS MEDICAL SYSTEMS CORP, Tokyo, Japan). The TCP-EBUS bronchoscope has a $5.9 \mathrm{~mm}$ distal tip, $5.7 \mathrm{~mm}$ insertion tube, $170^{\circ}$ angle of flexion and $20^{\circ}$ direction of view as compared to that of the CP-EBUS bronchoscope (6.9 $\mathrm{mm}, 6.3 \mathrm{~mm}, 120^{\circ}$ and $35^{\circ}$ ) and standard flexible bronchoscope $\left(5.5 \mathrm{~mm}, 5.1 \mathrm{~mm}, 210^{\circ}\right.$ and $0^{\circ}$ ), respectively (see Table 1). Figure 1 shows the CP-EBUS bronchoscope next to the TCP-EBUS bronchoscope. Each of the three bronchoscopes (without needles in the working channel) was then inserted sequentially into the following locations: left upper lobe (LUL), left lower lobe (LLL), right upper lobe (RUL), right middle lobe (RML), and right lower lobe (RLL). The number of bronchial generations accessible starting with the carina as the first was recorded. Fluoroscopy was performed at the most distal generation. The cadavers and fluoroscopy arm were kept stationary without change in position throughout the entire procedure.

A barium polymer was created using 5\% (by weight) barium sulfate in powder form added to an aqueous solution of 10\% (by weight) bovine skin gelatin (Sigma-Aldrich, St. Louis, MO) and 2\% agar (Sigma-Aldrich, St. Louis, $\mathrm{MO}$ ) and heated to $90^{\circ} \mathrm{C}$ during mixing and was maintained as a solution at $45-50^{\circ} \mathrm{C}$ prior to injection. Approximately $3 \mathrm{ml}$ of the polymer was then instilled into cadavers in a third-generation airway using a therapeutic bronchoscope (Olympus BF-1TH190) and Guide Sheath (Olympus K-203). The airways chosen to place the polymer were based on locations that are difficult to reach with conventional CP-EBUS. The barium polymer was placed in the anterior segment of the RUL (RB3) in the male cadaver and lateral basal segment of LLL (LB9), anterior segment of RUL (RB3), medial segment of RML (RB5) and medial basal segment of RLL (RB7) in the female cadaver. The TCP- EBUS bronchoscope was then inserted into the airways and used to observe the lesion endobronchially, as well as assess it via ultrasound if possible. A 22-gauge Olympus vizishot needle was deployed into the lesion to observe access with EBUS. The purpose of placing the polymer in the airway to was to view it endobronchially. Assessing sample obtained from the polymer was beyond the scope of this study.

The institutional review board at the Medical University of South Carolina approved this study (Pro\# 37817).

\section{Results}

The bronchoscopist for this study was unblinded and performed bronchoscopy with all three bronchoscopes on both the female and male cadaver with the knowledge of the purpose of the study and the difference between each of the three bronchoscopes. In the female cadaver, the standard flexible bronchoscope reached three generations of bronchi in the LUL, LLL, RUL and RML. In the RLL, the fourth-generation bronchus was reached. The standard EBUS bronchoscope reached one fewer generation than the standard bronchoscope at all locations (second generation in the LUL, LLL, RUL, and RML and third generation in the RLL). TCP-EBUS bronchoscope accessed the same distal generation of bronchi as the standard flexible bronchoscope (see Table 2). 
Table 2: The first and second bifurcations are set at the carina and bilateral second carina

\begin{tabular}{|c|c|c|c|}
\hline \multicolumn{4}{|c|}{ Number of Bifurcations Accessible by CP-EBUS, Thin EBUS and Standard } \\
\hline \multirow[t]{2}{*}{ Bronchoscopes } & CP-EBUS & Thin EBUS & Standard \\
\hline & Bronchoscope & Bronchoscope & Bronchoscope \\
\hline \multicolumn{4}{|l|}{ Female Cadaver } \\
\hline LUL & 2 & 3 & 3 \\
\hline LLL & 2 & 3 & 3 \\
\hline RUL & 2 & 3 & 3 \\
\hline RML & 2 & 3 & 3 \\
\hline RLL & 3 & 4 & 4 \\
\hline \multicolumn{4}{|l|}{ Male Cadaver } \\
\hline LUL & 1 & 3 & 3 \\
\hline LLL & 2 & 2 & 3 \\
\hline RUL & 2 & 3 & 3 \\
\hline $\mathrm{RML}^{*}$ & - & - & - \\
\hline RLL & 3 & 4 & 4 \\
\hline
\end{tabular}

In the male cadaver, the standard flexible bronchoscope reached the third generation bronchi in the LUL, LLL, and RUL and the fourth generation bronchi in the lingula and RLL. The RML could not be evaluated secondary to lung collapse. The standard EBUS reached the first generation bronchus in the LUL, the second generation bronchi in the LLL and RUL, and the third generation bronchi in the RLL and lingula. TCP-EBUS bronchoscope was navigated distally to the fourth generation bronchi in the RLL and lingula, third generation bronchi of the LUL and RUL, and the second generation LLL bronchus. Figure 2 shows fluoroscopic images obtained with each of the three scopes in both the male and female cadavers.

Barium polymer was placed in the lateral basilar segment of the LLL (LB9), anterior segment of the RUL (RB3), medial segment of the RML (RB5) and medial basilar segment of the RLL (RB7) in the female cadaver and anterior segment of RUL (RB3) in the male cadaver (see Figure 3). TCP-EBUS bronchoscope accessed each of the areas and needle biopsies were taken. The 22-gauge Olympus Vizishot needle is shown deployed into the polymer under EBUS (see Figure 4). The sample from these biopsies was not assessed for adequacy.

\section{Discussion}

The use of standard bronchoscopy to biopsy pulmonary lesions has a wide range of reported diagnostic yields, depending on the technique, lesion location, or lesion size. The addition of navigational bronchoscopy and $r$-EBUS may increase diagnostic yield, however, these modalities lack the ability to visualize a target in real-time during biopsy.9.10 CP-EBUS with real-time capability has revolutionized yield for centrally located lymph nodes and pulmonary lesions. ${ }^{3}$ To extend real-time sampling capability to the periphery of the lung has the potential to increase diagnostic yield in bronchoscopy. This study extends the work previously performed in a porcine model by showing the extended reach in cadaver subjects with a view both endoscopically and by fluoroscopy. ${ }^{7}$
Figure 2: Fluoroscopic comparison of the most distal subsegmental bronchi accessed

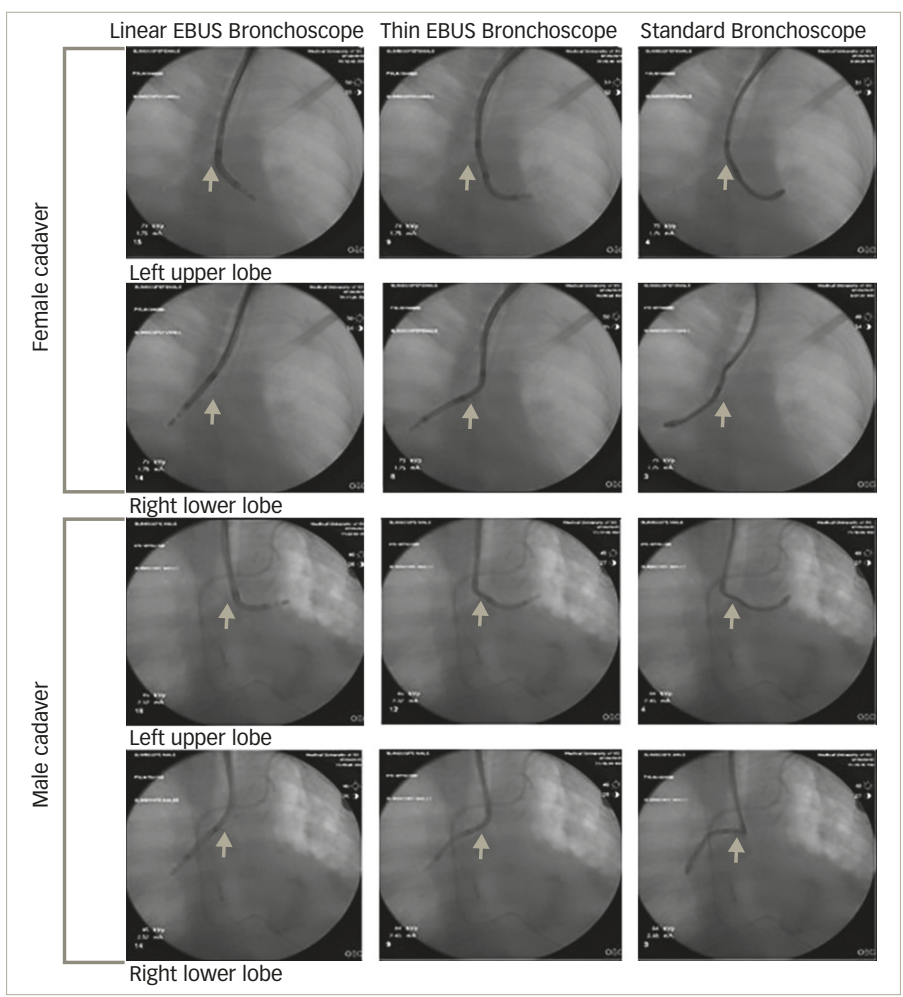

Gray arrow indicates the carina.

The TCP-EBUS prototype demonstrated improved accessibility of distal airways when compared to CP-EBUS bronchoscope. In both female and male cadaveric lungs, the TCP-EBUS bronchoscope accessed an additional generation and in most instances accessed the same distal airways as a standard bronchoscope. In addition, the specification improvements made in the TCP-EBUS bronchoscope allow for better access to more peripheral airways while maintaining an endoscopic view comparable to the CP-EBUS bronchoscope. The improved angulation allows for better maneuverability into upper lobes (most common location for lung cancer) as evidenced by the fact that lesions placed in the upper lobe were easily biopsied in real-time.

In the evaluation of pulmonary lesions suspected to be cancer, the American college of Chest Physicians (ACCP) guidelines recommend choosing a diagnostic modality based on the size, location, procedure risk, and available expertise." Historically, the sensitivity for standard bronchoscopy for diagnosing peripheral pulmonary lesions has been low at $34 \%$ and $63 \%$, for lesions $<2 \mathrm{~cm}$ or $>2 \mathrm{~cm}$ in diameter, respectively. ${ }^{11}$ In contrast, computed tomography-guided transthoracic needle biopsy has a relatively high sensitivity at $90 \% .{ }^{11}$

The use of guided bronchoscopy for diagnosis has become more appealing than standard bronchoscopy by offering promising techniques such as r-EBUS, virtual bronchoscopy software, and electromagnetic navigation (EMN). A meta-analysis of guided bronchoscopy for the evaluation of pulmonary nodules demonstrated a diagnostic yield of $70 \%{ }^{9}$ More recently, however, a publication from the ACCP Quality Improvement Registry, Evaluation and Education Program (AQuRE) reviewed 581 
Figure 3: Polymer instilled into the anterior segment of the right upper lobe in the female cadaver

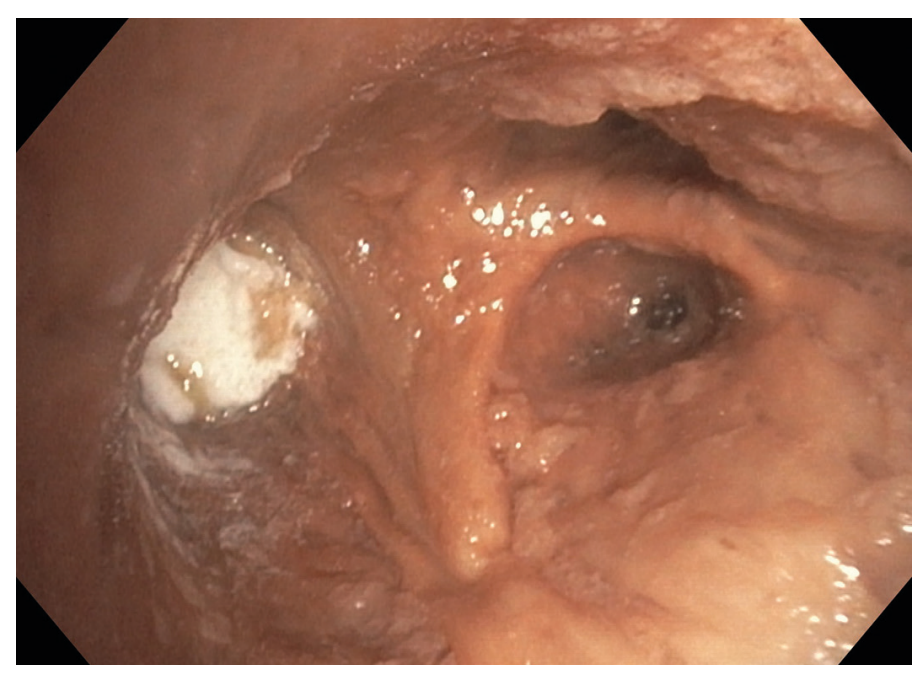

consecutive patients at 15 centers undergoing transbronchial biopsy (TBBX) for peripheral lesions and found an overall diagnostic yield of $53.7 \% .^{10}$ In that study, the diagnostic yield of standard bronchoscopy alone was $63.7 \%$, while the yield of r-EBUS, EMN, and EMN plus r-EBUS were lower (57\%, 38.5\%, and $47.1 \%$, respectively). ${ }^{10} \mathrm{~A}$ reason for the lower yield by the guided techniques may be related to case selection with more difficult cases being attempted by these techniques. In addition, a study evaluating a genomic classifier to predict malignancy in nodules and masses found the overall diagnostic yield of bronchoscopy was only $47 \% .{ }^{12}$ These findings suggest that current bronchoscopic techniques are not performing as well as necessary to diagnose pulmonary pathology.

CP-EBUS has the benefit of real-time sampling of lymph nodes and centrally located pulmonary lesions. The sensitivity of CP-EBUS is consistently high at $89 \%$ for mediastinal sampling and is recommended as the first diagnostic and staging test in suspected lung cancer. ${ }^{3}$ However, the technology is limited by its inability to reach more distal lesions (outside the central airways) due to its size and difficulty accessing upper lobe regions. While r-EBUS can access distal airways, the inability to biopsy in real-time and decreased performance on central lesions limits this technology. The TCP-EBUS represents a hybrid technology, which may overcome the limitations of both EBUS technologies, and may limit the number of bronchoscopes needed by sampling the mediastinum, and if necessary, the primary tumor in one setting, The ability to combine these techniques has been shown to decrease the overall number of procedures patients undergo during the work-up of a pulmonary nodule. ${ }^{8}$ The ability to access additional
Figure 4: Needle visualized with endobronchial ultrasound shown deployed into the polymer

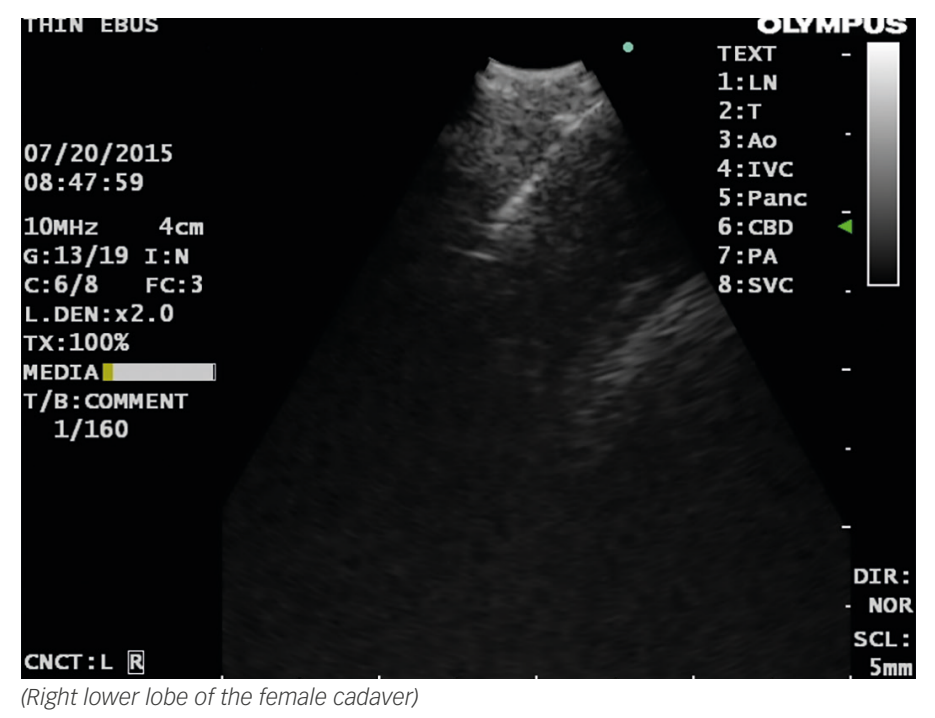

generations of airways may allow real time biopsy to be extended beyond the central airways and to potentially improve diagnostic yield of pulmonary nodules and masses. Although this technology appears to provide access to areas out of reach of conventional EBUS bronchoscope, it will not reach the most peripheral of pulmonary lesions that can be accessed via radial or EMN. However, this technology is an important step toward the development of an even thinner CP-EBUS bronchoscope that will extend further to the periphery.

This study has several limitations. First, the cadavers were fixed in an embalming solution and obviously rigor had set in which likely limited the extent to which any of the bronchoscopes could be passed to the periphery. More accurate measurements from the central airways may be accomplished once the bronchoscope is trialed in patients. It is unclear from this study how close to the pleural surface this technology can reach. Second, the bronchoscopists were not blinded to the location of the implanted polymer so that any assumptions regarding clinical utility are limited.

\section{Conclusion}

The TCP-EBUS bronchoscope can extend further into the distal airways than the traditional CP-EBUS bronchoscope with a reach similar to a standard bronchoscope. This technology has the potential to bring real-time biopsy capability to areas of the lung that currently cannot be reached by standard CP-EBUS. The goal being that if distal lesions can be biopsied in real time, then the yield of bronchoscopy should be substantially improved. Further evaluation in patients with pulmonary nodules and masses is warranted. $\square$
1. Gould MK, Donington J, Lynch WR, et al., Evaluation of individuals with pulmonary nodules: when is it lung cancer? Diagnosis and management of lung cancer, 3rd ed: American College of Chest Physicians evidence-based clinical practice guidelines, Chest, 2013;143:e93S-120S.

2. Ferlay J, Soerjomataram I, Dikshit R, et al., Cancer incidence and mortality worldwide: sources, methods and major patterns in GLOBOCAN 2012, Int I Cancer, 2015;136:E359-386.

3. 3. Silvestri GA, Gonzalez AV, Jantz MA, et al., Methods for staging non-small cell lung cancer: Diagnosis and management of lung cancer, 3rd ed: American College of Chest Physicians evidencebased clinical practice guidelines, Chest, 2013;143:e211S-250S.

4. Gupta D, Dadhwal DS, Agarwal R, et al., Endobronchial ultrasoundguided transbronchial needle aspiration vs conventional transbronchial needle aspiration in the diagnosis of sarcoidosis, Chest, 2014;146:547-56.

5. Grosu HB, lliesiu M, Caraway NP, et al.. Endobronchial Ultrasound Guided Transbronchial Needle Aspiration Accurately Diagnoses and Subtypes Lymphoma, Ann Am Thorac Soc, 2015;12(9):1336-44.

6. Kang HJ, Hwangbo B, Technical aspects of endobronchial ultrasound-guided transbronchial needle aspiration, Tuberc Respir Dis (Seoul), 2013;75:135-9.

. Wada H, Hirohashi K, Nakajima T, et al., Assessment of the new thin convex probe endobronchial ultrasound bronchoscope and the dedicated aspiration needle: a preliminary study in the porcine lung, I Bronchology Interv Pulmonol, 2015;22:20-7.

8. Yarmus $L$, Akulian J, Ortiz R, et al., A randomized controlled trial evaluating airway inspection effectiveness during endobronchial ultrasound bronchoscopy, J Thorac Dis, 2015;7:1825-2.

9. Memoli JS, El-Bayoumi E, Pastis NJ, et al., Using endobronchial ultrasound features to predict lymph node metastasis in patients with lung cancer, Chest, 2011;140:1550-6.

10. Ost DE, Ernst A, Lei $X$, et al., Diagnostic Yield and Complications of Bronchoscopy for Peripheral Lung Lesions: Results of the AQUIRE Registry, Am J Respir Crit Care Med, 2016 Jan 1;193(1):68-77.

11. Rivera MP, Mehta AC, Wahidi MM, Establishing the diagnosis of lung cancer. Diagnosis and management of lung cancer, 3rd ed American College of Chest Physicians evidence-based clinical practice guidelines, Chest, 2013;143:e142S-165S.

12. Silvestri $G A$, Vachani $A$, Whitney $D$, et al., $A$ Bronchial Genomic Classifier for the Diagnostic Evaluation of Lung Cancer, N Eng/ J Med, 2015;373:243-51. 